\title{
Fission-barrier heights of neutron-deficient mercury nuclei
}

\author{
M. Veselský,,${ }^{1, *}$ A. N. Andreyev, ${ }^{2}$ S. Antalic, ${ }^{3}$ M. Huyse, ${ }^{4}$ P. Möller, ${ }^{5}$ K. Nishio, ${ }^{6}$ A. J. Sierk,${ }^{5}$ \\ P. Van Duppen, ${ }^{4}$ and M. Venhart ${ }^{1,4}$ \\ ${ }^{1}$ Institute of Physics, Slovak Academy of Sciences, 84511 Bratislava, Slovakia \\ ${ }^{2}$ School of Engineering, University of the West of Scotland, Paisley, PAI 2BE, United Kingdom, \\ and the Scottish Universities Physics Alliance (SUPA) \\ ${ }^{3}$ Department of Nuclear Physics and Biophysics, Comenius University, 84248 Bratislava, Slovakia \\ ${ }^{4}$ Instituut voor Kern- en Stralingsfysica, KU Leuven, Celestijnenlaan 200D, 3001 Leuven, Belgium \\ ${ }^{5}$ Theoretical Division, Los Alamos National Laboratory, Los Alamos, New Mexico 87545, USA \\ ${ }^{6}$ Advanced Science Research Center, Japan Atomic Energy Agency, Tokai, Ibaraki 319-1195, Japan
}

(Received 25 June 2012; published 15 August 2012)

\begin{abstract}
The recently measured probabilities of the $\beta$-delayed fission for ${ }^{178,180} \mathrm{Tl}$ are used to deduce the fission-barrier heights of the daughter isotopes ${ }^{178,180} \mathrm{Hg}$, undergoing low-energy fission. Four alternative $\beta$-decay strength functions and four variants of the statistical model of de-excitation of the daughter nucleus are used to determine the fission-barrier height for ${ }^{180} \mathrm{Hg}$. Depending on the choice of the model, the deduced fission-barrier heights appear to be between $10 \%$ and $40 \%$ smaller than theoretical estimates. This observation is verified also for fission-barrier heights extracted using the probability of $\beta$-delayed fission of ${ }^{178} \mathrm{Tl}$. The spread in extracted fission-barrier heights results mainly from uncertainties in the magnitude of the pairing gap at the saddle configuration.
\end{abstract}

DOI: 10.1103/PhysRevC.86.024308

PACS number(s): 25.85.-w, 27.70.+q, 23.40.-s, 24.60.Dr

\section{INTRODUCTION}

Historically, different approaches have been used to deduce fission-barrier heights, as documented in extensive reviews $[1,2]$. One of them, specifically useful for very neutrondeficient nuclei in the mass region of $A>150$, involves a comparison of the experimental production cross section of evaporation residues with results of statistical model calculations, used to estimate the survival probability of the compound nuclei during the de-excitation cascade [3-12]. A general conclusion from such high-energy fission studies, where the excitation energy of the fissioning nucleus $\left(E^{*}=\right.$ $30-150 \mathrm{MeV}$ ) is much larger than the expected fission barrier, is that the liquid-drop fission barrier decreases faster with increasing neutron deficit than given by commonly used theoretical predictions. For example, for the most neutrondeficient isotopes in the lead region [5-12], the theoretical fission-barrier heights [13-15] are about $20 \%$ to $30 \%$ lower than the experimental ones. This is an interesting observation since when using the available experimental values of the ratio $\Gamma_{\mathrm{f}} / \Gamma_{\mathrm{n}}$, being the ratio of decay widths of fission and neutron emission, for nuclei close to the $\beta$-stability line [16], the statistical approach leads to a smaller reduction of theoretical fission barriers by only about $5-10 \%$. This suggests that the magnitude of the observed deviation is influenced by the isospin asymmetry of the fissioning nuclei.

In this respect, the process of $\beta$-delayed fission $(\beta \mathrm{DF})$, recently experimentally observed in the region of neutrondeficient isotopes between thalium and francium, offers unique possibilities to deduce fission barriers and to investigate the reduction in an independent way. Most notably, fission following the $\beta$ decay of a parent nucleus occurs at low excitation energy

*martin.veselsky@savba.sk and angular momentum and allows one to probe the fission barrier under such favorable conditions. The values of fissionbarrier heights, which can be determined by this analysis, are also relevant for the efforts to synthesize superheavy nuclei and exotic proton-rich nuclei for spectroscopic investigations, where the statistical model of de-excitation is widely used to predict the production cross sections.

$\beta$-delayed fission, discovered in 1966 in Dubna for the isotopes ${ }^{232,234} \mathrm{Am}$ [17] and first correctly interpreted in [18], is a two-step process. In the first step, a parent nucleus undergoes $\beta$ decay, populating excited states in the daughter nucleus, up to the maximum excitation energy defined by the available $\mathrm{Q}_{\mathrm{EC}}$ value. In the second step, if excited states of the daughter nuclide are populated around or above the fission barrier $\mathrm{B}_{f}$, the nucleus can undergo decay by fission, which competes with the emission of $\gamma$ quanta down to the ground state. Due to the large neutron deficit of the fissioning daughter nucleus, for example, $N / Z=1.25$ for the case of ${ }^{180} \mathrm{Hg}$ considered in this paper, no neutron emission typically contributes due to high neutron-separation energy. This simplifies the analysis in comparison with the analysis of data closer to the stability line. $\beta$-delayed fission can be observed for extremely neutron-deficient nuclei, where the necessary condition of $Q_{\mathrm{EC}}$ (parent) $\simeq B_{\mathrm{f}}$ (daughter) is fulfilled. On the neutron-rich side, $\beta$-delayed fission constitutes, together with spontaneous and neutron-induced fission, an obstacle for the production of heavy nuclei in the astrophysical $r$ process.

$\beta$-delayed fission is of special interest because it allows one to study the low-energy fission properties (e.g., decay probability, fission-barrier height, mass and charge distributions, total kinetic energy, $\gamma$ and neutron multiplicities) of exotic neutron-deficient daughter nuclei that have no observable spontaneous fission branch in their ground state. Fission following $\beta$ decay of the parent nucleus offers the possibility 
of studying the fission process at both low excitation energy and low (zero) angular momentum of the fissioning nucleus and thus provides an alternative to the study of high-energy fission in complete fusion. In particular, nonzero value of the angular momentum is supposed to reduce the fission barrier. However, the rotating-liquid-drop models of Cohen, Plasil, and Swiatecki [14] and Sierk [15] estimate that such an effect would play a significant role only for the values of angular momentum above $10 \hbar$, which are not reached in the process of $\beta$ decay, and thus the approximation of zero angular momentum appears to be justified.

Experimentally, the probability of $\beta$-delayed fission, $P_{\beta \mathrm{DF}}$, is defined as the ratio of the observed number of $\beta$ decays followed by fission, $N_{\beta \mathrm{DF}}$, to the total number of observed $\beta$ decays, $N_{\beta}$. In this work, the fission-barrier height for the daughter nuclides ${ }^{178,180} \mathrm{Hg}$ will be deduced by comparing the experimental probabilities of $\beta$-delayed fission for ${ }^{178,180} \mathrm{Tl}$, which was recently measured at ISOLDE in CERN [19], to the values obtained using statistical-model predictions for deexcitation.

\section{PROBABILITY OF $\beta$-DELAYED FISSION}

The probability of $\beta$-delayed fission can be determined [20] using the expression:

$$
P_{\beta \mathrm{df}}=\frac{\int_{0}^{\mathrm{Q}_{\beta}} F\left(Q_{\beta}-E\right) S_{\beta}(E) \frac{\Gamma_{\mathrm{f}}(E)}{\Gamma_{\mathrm{f}}(E)+\Gamma_{\gamma}(E)} d E}{\int_{0}^{\mathrm{Q}_{\beta}} F\left(Q_{\beta}-E\right) S_{\beta}(E) d E},
$$

where $F\left(Q_{\beta}-E\right)$ is the statistical Fermi function, $S_{\beta}(E)$ is the $\beta$-strength function, $\Gamma_{\mathrm{f}}$ is the fission width, and $\Gamma_{\gamma}$ is the $\gamma$ decay width. Typically, only the two most dominant channels, that is, fission and $\gamma$-ray emission, are considered at excitation energies below $Q_{\mathrm{EC}}$. Emission of neutrons is hindered by the high neutron separation energy in the neutron-deficient nuclei (values of $Q_{\mathrm{EC}}$ for ${ }^{180} \mathrm{Tl}$ and $S_{\mathrm{n}}$ for ${ }^{180} \mathrm{Hg}$ are $10.99 \mathrm{MeV}$ and $11.40 \mathrm{MeV}$ [21], respectively). The emission of protons, which have a lower separation energy than neutrons in the neutron-deficient nuclei, is hindered because of the Coulomb barrier.

The fission width of the excited nucleus can be expressed [22]

$$
\begin{aligned}
\Gamma_{\mathrm{f}}\left(E^{*}\right)= & \frac{1}{2 \pi \rho_{\mathrm{c}}\left(E^{*}-\Delta\right)} \\
& \times \int_{0}^{E^{*}-B_{\mathrm{f}}-\Delta_{\mathrm{sp}}} \rho_{\mathrm{sp}}\left(E^{*}-B_{\mathrm{f}}-\Delta_{\mathrm{sp}}-E^{\prime}\right) d E^{\prime},
\end{aligned}
$$

where $\rho_{\mathrm{c}}\left(E^{*}-\Delta\right)$ and $\rho_{\mathrm{sp}}\left(E^{*}-B_{\mathrm{f}}-\Delta_{\mathrm{sp}}-E^{\prime}\right)$ are level densities of the excited intermediate nucleus after $\beta$ decay and of its deformed saddle configuration, respectively, $B_{\mathrm{f}}$ is the fission-barrier height, $\Delta$ is the pairing gap causing a sharp cutoff in the level density of the daughter nucleus, and $\Delta_{\mathrm{sp}}$ is the pairing gap of the fissioning nucleus in its saddle configuration. The level density of the nucleus with excitation energy $E^{*}$ can be expressed within the framework of the Fermi-gas model, with the level-density parameter $a_{\mathrm{n}}$ (the index $\mathrm{n}$ is introduced to distinguish it from the level density at the saddle configuration $a_{\mathrm{f}}$ ) taken according to the formula of
Ignatyuk [23], which takes into account the shell correction to the ground-state mass and its damping with excitation energy by the constant $E_{\mathrm{d}}=18.5 \mathrm{MeV}$, leading to the asymptotic value $\tilde{a}_{\mathrm{n}}$ at high excitation energy. For the saddle configuration, no shell correction is assumed and therefore the level density in the saddle configuration $a_{\mathrm{f}}$ is fixed at a constant value $a_{\mathrm{f}}=\tilde{a}_{\mathrm{n}}$. In analogy to commonly used statistical codes for de-excitation of highly excited nuclei, sub-barrier fission is not considered.

As described in Refs. [5-11], the statistical model for the decay of a compound nucleus combines the expression for $\Gamma_{\mathrm{f}}$ given by Eq. (2) with Hauser-Feshbach expressions for the emission widths of neutron, proton, and $\alpha$ particles. In order to reproduce the observed evaporation-residue cross sections for neutron-deficient nuclei at excitation energies varying from 30 up to $150 \mathrm{MeV}$, a reduction of theoretical fission barriers by 20$30 \%$ is necessary. Detailed analysis of dependence of $\Gamma_{f} / \Gamma_{n}$ of neutron-deficient Ra nuclei as a function of excitation energy [10] established that the reduction of fission barrier using a fixed level-density parameter in the fission channel $\left(a_{\mathrm{f}}=\tilde{a}_{\mathrm{n}}\right)$ is preferred over the alternative possibility to enlarge $a_{\mathrm{f}}$ over $\tilde{a}_{\mathrm{n}}$ by $5-10 \%$. Moreover, compared to compound-nucleus fission, $\beta$-delayed fission occurs at low excitation energies and thus the eventual variation of $a_{\mathrm{f}}$ with respect to $\tilde{a}_{\mathrm{n}}$ practically does not influence the resulting fission probabilities.

The $\gamma$-decay width $\Gamma_{\gamma}$ can be determined using the empirical formula of Stolovy and Harvey, see Ref. [24], which is based on experimental $\gamma$-width data of heavy nuclei and therefore can be considered as a reasonable estimate of the widths for the $\gamma$ emission at excitation energies up to $10 \mathrm{MeV}$. It was introduced as

$$
\Gamma_{\gamma}\left(E^{*}\right)=0.00053 A^{2 / 3} D\left(E^{*}-\Delta\right)^{0.25}\left(E^{*}-\Delta\right)^{4.3}[\mathrm{meV}] \text {, }
$$

where the level spacing $D\left(E^{*}-\Delta\right)$ is given in units of electron volts, the excitation energy is given in million electron volts, and the result is obtained in million electron volts. The level spacing is defined as $D\left(E^{*}-\Delta\right)=\rho^{-1}\left(E^{*}-\Delta\right)$. Here, $\rho\left(E^{*}-\Delta\right)$ can be calculated using the empirical formula of Gilbert and Cameron; see Ref. [25].

Combining formula (1) with the model framework presented above allows us to determine the fission-barrier height $B_{\mathrm{f}}$ from the experimental value of $\beta$-delayed fission probability $P_{\beta \mathrm{df}}$, provided a proper $\beta$-strength function $S_{\beta}$ is used.

\section{FISSION-BARRIER HEIGHTS OF THE ISOTOPES ${ }^{178,180} \mathrm{Hg}$}

In recent experiments performed at ISOLDE (CERN)—see the detailed discussion in Refs. [19,26,27]-a $\beta$-delayed fission probability $P_{\beta \mathrm{df}}=3.2(2) \times 10^{-5}$ was deduced for ${ }^{180} \mathrm{Tl}$ and $P_{\beta \mathrm{df}}=1.5(6) \times 10^{-3}$ for ${ }^{178} \mathrm{Tl}$. We used these values to determine the fission-barrier height of daughter mercury isotopes ${ }^{178,180} \mathrm{Hg}$. Previously (see Ref. [28]) the fission barrier of ${ }^{180} \mathrm{Hg}$ was extracted from the $\beta$-delayed fission probability of $P_{\beta \mathrm{df}}\left({ }^{180} \mathrm{Tl}\right)=10^{(-7 \pm 1)}$ reported by Lazarev et al. [29-31], and the probability of $\beta$-delayed fission reported there is by up to two orders of magnitude smaller than the value reported in Refs. [19,26]. However, now it is clear 
that the Lazarev's value was underestimated; see discussion in Ref. [26].

To determine the sensitivity of extracted fission-barrier height to different model assumptions, we use different approaches and parametrizations for the crucial constituents in formula (1), namely the $\beta$-strength function $S_{\beta}$, which characterizes the distribution of excited intermediate states produced after $\beta$ decay, and level densities and pairing interaction at the intermediate state and at the saddle point. The range of the used approximations and alternative model parameters was made deliberately wide in order to cover a range of possible alternatives.

\section{A. $\beta$-strength function $S_{\beta}$}

In the present work, we used four different $\beta$-strength functions for ${ }^{180} \mathrm{Tl}$. Two of them, which are given in Figs. 1(a) and 1(b), were calculated using a Quasi-Particle Random Phase Approximation (QRPA) approach [34] with assumed deformation of either the parent or daughter nucleus (in further text denoted as [34]-p.d. and [34]-d.d., respectively). It is necessary to test the sensitivity of the obtained results to the assumed shape of the parent or daughter nucleus, as shape coexistence has been observed in this region; see, for example, works [35] and references therein. While the parent isotope ${ }^{180} \mathrm{Tl}$ is most probably nearly spherical $\left(\epsilon_{2} \sim\right.$ -0.05) [36], the daughter nucleus ${ }^{180} \mathrm{Hg}$ exhibits coexistence of weakly $\left(\epsilon_{2} \sim-0.13\right)$ and strongly deformed $\left(\epsilon_{2} \sim 0.26\right)$ $0^{+}$configurations $[35,37,38]$. For comparison, the calculated $\beta$-strength function taken from Ref. [28] was also used [see Fig. 1(c)]. In all the calculated $\beta$-strength functions shown in Fig. 1, only a small part of $\beta$ strength feeds states close to the top of predicted $\mathrm{B}_{\mathrm{f}}$, which can be one of the main reasons for small $\mathrm{P}_{\beta \text { df }}$ for ${ }^{180} \mathrm{Tl}$. Finally, a simple assumption of a flat $\beta$-strength function was investigated as well. The assumption of a flat $\beta$-strength function provides a simplified structureless approximation of the experimental $\beta$-strength function and, in comparison with the microscopic $\beta$-strength functions, allows one to judge the effect that the presence of a specific structure-driven effect in the $\beta$-strength function can have on the final value of the fission-barrier height. The flat $\beta$-strength function also provides a simple tool to compare model parameters in the prediction of $\beta$-delayed fission of various nuclei and it was used for analysis of the very recently observed $\beta$-delayed fission of ${ }^{178} \mathrm{Tl}$; see below.

\section{B. Level-density formula for the intermediate nucleus and saddle configuration}

Apart from the choice of the $\beta$-strength function, several possibilities exist also concerning the choice of level-density formula for the intermediate nucleus after $\beta$ decay and its saddle configuration, specifically concerning the way the pairing interaction is introduced. It is usually assumed that pairing leads to a gap in the level densities above the ground state, which is reflected by a cutoff in excitation energy $\left(U=E^{*}-\Delta\right)$. Nevertheless, it is not clear whether the pairing
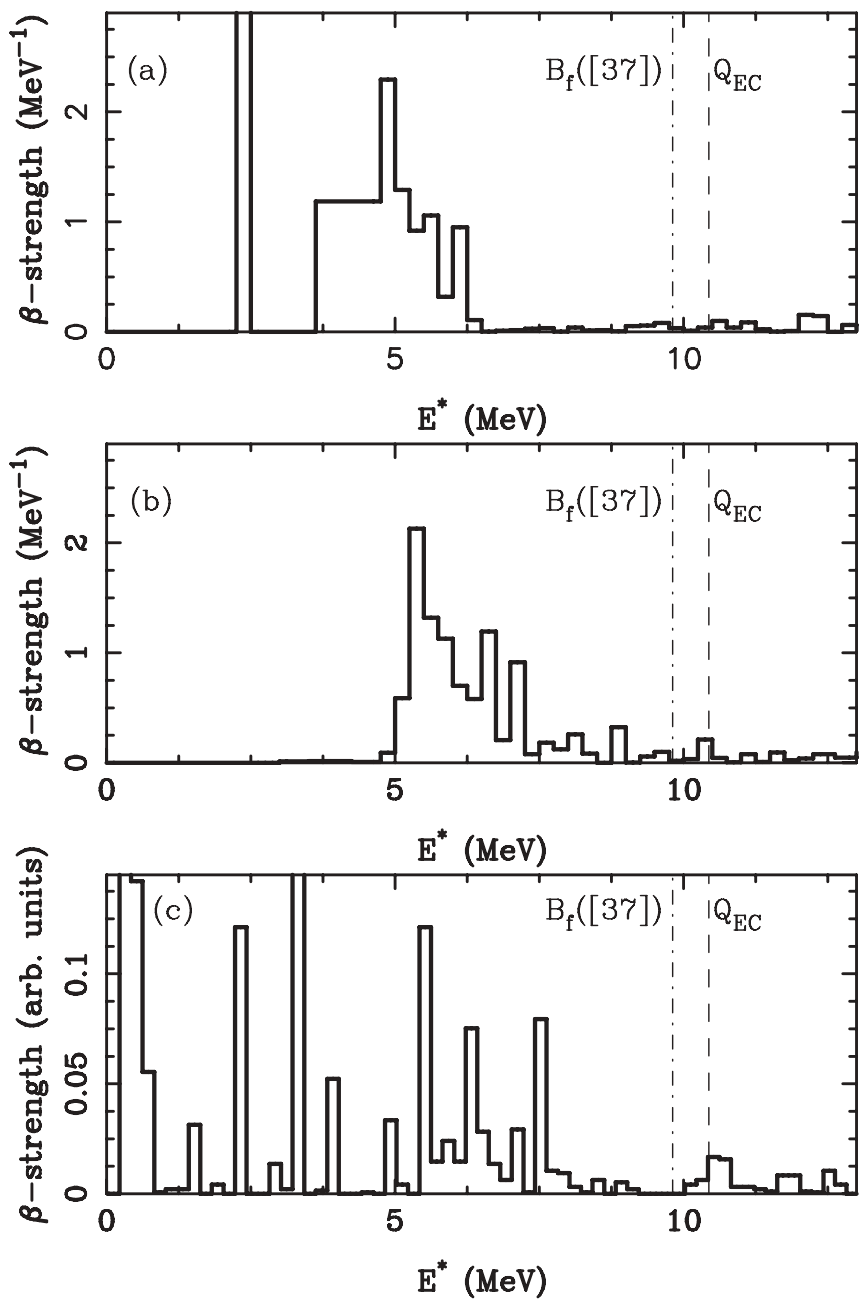

FIG. 1. Calculated $\beta^{+} / \mathrm{EC}$-strength functions for ${ }^{180} \mathrm{Tl}$ decay, calculated as in [34] with assumed deformation of (a) ${ }^{180} \mathrm{Tl}$ (parent nucleus) and (b) ${ }^{180} \mathrm{Hg}$ (daughter nucleus). In the text, these $\beta^{+} / \mathrm{EC}$ strength functions are denoted as [34]-p.d. and [34]-d.d., respectively. (c) $\beta^{+} /$EC-strength function taken from Ref. [28]. Note that authors of Refs. [34] and [28] used in their original publication different units for the $\beta^{+} / \mathrm{EC}$ strength, which accounts for the differences in panels (a), (b), and (c). Dashed vertical lines represent the calculated value of $Q_{\text {EC }}$ for ${ }^{180} \mathrm{Tl}$ decay from [32] and dash-dotted vertical lines represent the calculated value of the fission-barrier height for ${ }^{180} \mathrm{Hg}$ [33].

gap in the saddle configuration $\Delta_{\text {sp }}$ is equal to its value in the ground state $\Delta$. One can define two extreme possibilities, either pairing gap in the saddle remains identical to the ground state $\left(\Delta_{\mathrm{sp}}=\Delta\right)$ or it fully vanishes $\left(\Delta_{\mathrm{sp}}=0\right)$. As a first choice the level-density formula of Ignatyuk [23], which is based on the Fermi-gas formula to which it introduces the shell-structure effect, is used for the initial state, that is, the compound nucleus in the case of fusion-evaporation reaction or the daughter nucleus after $\beta$ decay. For the saddle configuration, in the absence of shell corrections, the formula of Ignatyuk is identical to the Fermi gas formula, where shell effects are not considered. Alternatively, one can use the formula of Gilbert and Cameron [25], which, as an exponential fit to the low-energy excitation spectrum, extends even to 

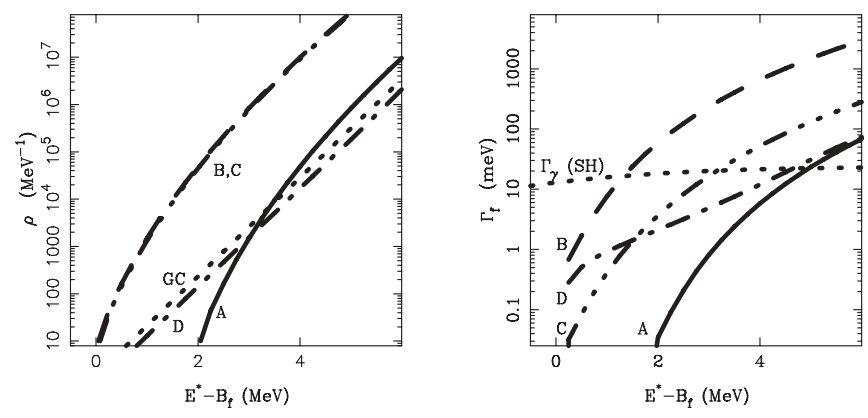

FIG. 2. (Color online) (a) Level densities at the saddle configuration of ${ }^{180} \mathrm{Hg}$ calculated for variants $\mathrm{A}-\mathrm{D}$, as described in the text. As a reference, the result of a calculation with the formula of Gilbert and Cameron for the initial state with the shell correction to the level-density parameter is shown as a dotted line denoted as GC. Note that in variants $\mathrm{B}$ and $\mathrm{C}$ the same level densities are used. (b) Fission widths of ${ }^{180} \mathrm{Hg}$ calculated for variants A-D (with fission-barrier height set arbitrarily to $8 \mathrm{MeV}$ ). For comparison, the dotted line denoted as SH gives $\gamma$-emission widths calculated using the formula of Stolovy and Harvey.

excitation energies below the expected value of the pairing gap. Based on the above, four approaches were investigated for the (even-even) nuclei ${ }^{178,180} \mathrm{Hg}$ :

(i) Variant A. Level-density formula of Ignatyuk [23] is used in the intermediate state populated by $\beta$ decay. The Fermi-gas formula is used in the saddle configuration and the pairing gap in the saddle configuration is fully preserved $\left(\Delta_{\mathrm{sp}}=\Delta\right)$.

(ii) Variant $B$. Same as variant $A$, but without a pairing gap at the saddle configuration $\left(\Delta_{\mathrm{sp}}=0\right)$.

(iii) Variant $C$. Without a pairing gap and thus using Fermigas formula for level density in both the intermediate state $(\Delta=0)$ and the saddle configuration $\left(\Delta_{\mathrm{sp}}=0\right)$.

(iv) Variant $D$. The level densities of Gilbert-Cameron [25] in both the initial state and the saddle configuration, while shell corrections to the level-density parameter are not applied in the saddle configuration.

Figure 2(a) gives the calculated level densities in the saddle configuration of ${ }^{180} \mathrm{Hg}$ that were obtained using the above variants. Labeled lines represent the level density calculated for a given excitation energy above the fission barrier. The effect of the level density on the fission widths of ${ }^{180} \mathrm{Hg}$ (with fission-barrier height set arbitrarily to $8 \mathrm{MeV}$ ) is shown in Fig. 2(b).

The above model assumptions concerning the level density at the saddle configuration essentially cover a range of possible results expected from the detailed microscopic calculations. The primary goal of this model analysis is to provide a guidance for a proper choice of parameters in the statistical description of the de-excitation of the compound nuclei. A realistic model assumption for the level density is crucial to achieve the necessary predictive power of calculations.

\section{Determination of the fission-barrier heights for ${ }^{178,180} \mathrm{Hg}$}

Table I and Fig. 3(a) give the extracted values of fissionbarrier heights of ${ }^{180} \mathrm{Hg}$, corresponding to the experimental value of $P_{\beta \mathrm{df}}=3.2(2) \times 10^{-5}$, for the four alternative $\beta$ strength functions and the four variants of level densities. For the calculation of the $\gamma$-emission width, the formula of Stolovy and Harvey [24] was used. The angular momentum was assumed to be zero since this approximation appears to be justified in the $\beta$-delayed fission of ${ }^{180} \mathrm{Tl}$, where the spin of $I=(4,5)$ was deduced in [35].

Since the extracted values of fission barriers represent total fission barriers, which can be interpreted as a sum of macroscopic and microscopic components, theoretical liquid-drop barriers from Refs. $[14,15]$ need to be modified by corresponding microscopic corrections. The macroscopic fission barriers of Cohen, Plasil, and Swiatecki [14] are typically corrected by subtracting the ground-state shell corrections, which are obtained as a difference of experimental mass and liquid-drop mass calculated using formula of Myers and Swiatecki [13], as it is done in the statistical code HIVAP [5]. The same approach is used in the HIVAP code also for fission barriers of Sierk [15]. However, since the model of Sierk starts from the spherical liquid-drop formula of Möller and Nix [39], it is appropriate to use this formula also for calculation of the corresponding ground-state shell correction. This prescription is used in this work. Using experimental masses from the 2011 update of the 2003 Atomic Mass Evaluation [21,40], $Q_{\mathrm{EC}}=10.99 \pm 0.06$ $\mathrm{MeV}$ was obtained for ${ }^{180} \mathrm{Tl}$ Electron Capture (EC) decay. The resulting uncertainty of the extracted fission-barrier height for a specific calculation is approximately $0.1 \mathrm{MeV}$ (up to $2 \%$ of total value), due to the uncertainty of used value of $Q_{\mathrm{EC}}$ (estimated in $\left.[21,40]\right)$ and uncertainty of the probability of $\beta$-delayed fission.

TABLE I. Extracted values of fission-barrier height of ${ }^{178,180} \mathrm{Hg}$.

\begin{tabular}{|c|c|c|c|c|c|c|c|c|c|}
\hline \multirow{3}{*}{${ }^{180} \mathrm{Hg}$} & [34]-d.d. & 7.23 & 8.96 & 8.38 & 8.62 & $8.1(9)$ & & & \\
\hline & [28] & 6.15 & 7.88 & 7.33 & 7.52 & $7.0(9)$ & & & \\
\hline & Flat & 7.11 & 8.84 & 8.20 & 8.36 & $8.0(9)$ & & & \\
\hline
\end{tabular}


(a) ${ }^{180} \mathrm{Hg}$

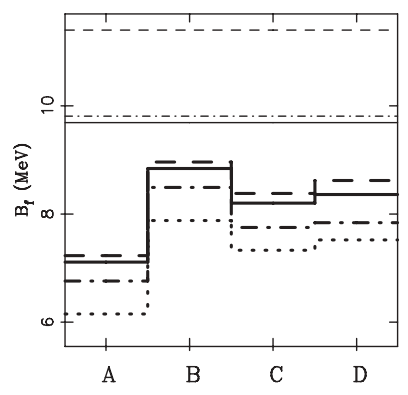

(b) ${ }^{178} \mathrm{Hg}$

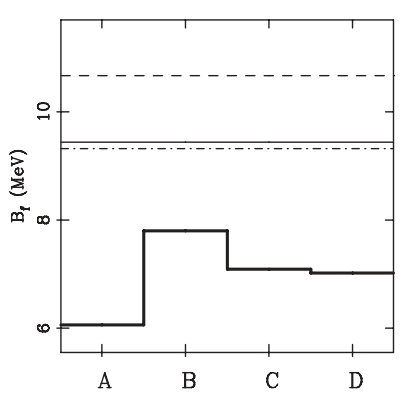

FIG. 3. (Color online) (a) Fission-barrier heights of ${ }^{180} \mathrm{Hg}$ in four variants $\mathrm{A}-\mathrm{D}$. Four $\beta$-strength functions were used: calculated in work [34] assuming deformation either of ${ }^{180} \mathrm{Tl}$ (red dashed line) or ${ }^{180} \mathrm{Hg}$ (green dash-dotted line), flat function (black solid line), and $\beta$-strength function taken from Ref. [28] (blue dotted line). The horizontal solid and dashed lines give theoretical values of fission-barrier heights, obtained as a sum of rotating-liquid-drop barriers of Sierk [15] (solid) and Cohen-Plasil-Swiatecki [14] (dashed) with their respective ground-state shell correction; see the text for details. Horizontal dash-dotted line gives the theoretical value taken from macroscopic-microscopic calculation by Möller [33]. (b) Same as panel (a) but for ${ }^{178} \mathrm{Hg}$. In this case only a flat $\beta$-strength function was used.

It is apparent that the extracted values of fission-barrier height are by $10 \%$ to $40 \%$ lower than the corresponding values based on rotating liquid-drop values of Sierk $\left(B_{\mathrm{f}}=9.69 \mathrm{MeV}\right)$ and Cohen, Plasil, and Swiatecki ( $\left.B_{\mathrm{f}}=11.40 \mathrm{MeV}\right)$, which are typically used in the statistical model calculations.

Out of the four $\beta$-strength functions, the calculation assuming the deformation of the parent nucleus ${ }^{180} \mathrm{Tl}$, that is, [34]-p.d. variant [dash-dotted line in Fig. 3(a)], leads to lower values of fission-barrier heights than the other $\beta$-strength function ( [34]-d.d.); see dashed line in Fig. 3(a). This is mostly due to absence of strength at excitation energies above $6.5 \mathrm{MeV}$ and larger strength at low energies. The $\beta$-strength function calculated assuming the deformation of the daughter nucleus ( [34]-d.d.) feeds more evenly in this region and leads to results close to that of the flat $\beta$-strength function [black solid line Fig. 3(a)]. The $\beta$-strength function taken from Ref. [28] [dotted line in Fig. 3(a)] leads to lowest values of fission-barrier heights, as a consequence of the $\beta$ feeding below excitation energy $1 \mathrm{MeV}$. The flat $\beta$-strength function appears to be a reasonable approximation in the case when the $\beta$-strength function changes smoothly. On the other hand, the deduced fission-barrier heights depend strongly on the pronounced structure of the $\beta$-strength function at excitation energies close to both the expected fission barrier and minimal excitation energy. The available structure information for mercury isotopes favors deformation of the daughter nucleus [35], and so it seems that the $\beta$-strength function calculated assuming the deformation of the daughter nucleus (denoted as [34]-d.d.) provides the best estimate of experimental data. As documented in Table I, the overall range of values resulting from different choices of the $\beta$-strength function amounts to about $\pm 0.6 \mathrm{MeV}$.

The behavior observed in Fig. 3 appears to be consistent with statistical calculations of evaporation-residue cross sections, where similar scaling of fission barriers is needed; see Refs. [3-12]. It is however remarkable how sensitive the extracted fission barriers are to the treatment of pairing in the saddle configuration. From the above variants of the calculation, variant A (with fully preserved pairing gap at the saddle point) is typically used in the statistical codes like HIVAP [5], leading to the reduction of theoretical predictions of fission barriers by 30-45\%. On the other hand, variant B, without a pairing gap in the saddle point, leads to reduction of fission-barrier heights by up to $30 \%$ of the total fissionbarrier height. The difference between fission-barrier heights extracted in variants $\mathrm{A}$ and $\mathrm{B}$ amounts up to $2 \mathrm{MeV}$ (about $20 \%$ of the fission-barrier height), which also defines the total uncertainty due to various choices of the level density and pairing gap (A-D), shown in Table I. Interpretation of the observed behavior depends crucially on proper treatment of the pairing gap in the saddle configuration. It seems natural to assume that pairing will be at least partially preserved even in the saddle configuration, and the recent calculation [33] appears to corroborate such an assumption. This suggests that the most probable value of the fission-barrier heights will be located between the two extreme values, obtained in the variants $\mathrm{A}$ and $\mathrm{B}$. Variant $\mathrm{C}$ differs from variant $\mathrm{B}$ by assuming zero pairing gap also at the ground state, which results in a drop of the fission barriers by more than $0.5 \mathrm{MeV}$. Variant $\mathrm{D}$ leads to fission-barrier heights similar to variant $\mathrm{C}$, despite different level-density formulas with different low-energy dependences [see Fig. 2(b)], thus demonstrating that it is the value of the pairing gap in the fission channel which influences most strongly the extracted fission-barrier height. As can be seen from comparing variants $C$ and $D$, sensitivity to the details of the level structure above the pairing gap is weaker. This suggests the plausibility of the statistical model of the compound nucleus de-excitation for the extraction of fission-barrier heights.

Besides ${ }^{180} \mathrm{Tl}, \beta$-delayed fission was observed recently for ${ }^{178} \mathrm{Tl}$ [27]. Using the flat $\beta$-strength function, which leads to comparable results with other $\beta$-strength functions in the case of ${ }^{180} \mathrm{Hg}$, we extracted fission-barrier heights for ${ }^{178} \mathrm{Hg}$, and the results are given in Table I and Fig. 3(b). In both cases, $Q_{\text {EC }}$ was calculated using masses from AME2011 [21,40] (leading to the values $Q_{\mathrm{EC}}=11.52 \pm 0.1 \mathrm{MeV}$ ). It is apparent that results for ${ }^{178} \mathrm{Hg}$ are similar for ${ }^{180} \mathrm{Hg}$. Again variant $\mathrm{A}$, assuming total preservation of the pairing in the saddle, leads to the largest reduction of $35-45 \%$ and variant $\mathrm{B}$, assuming total disappearance of the pairing in the saddle, to the smallest reduction of about 20-30\%, compared with the macroscopic values of Sierk [15] and Cohen, Plasil, and Swiatecki [14], corrected by corresponding ground-state shell correction as discussed above, or with the recent macroscopic-microscopic calculation of Möller et al. [33]. Again the most probable value of the fission barrier can be expected between the two extreme values, obtained in variants $\mathrm{A}$ and $\mathrm{B}$, and thus in fact is close to the values obtained in the variants $\mathrm{C}$ and D. Analogous analysis as for ${ }^{178} \mathrm{Hg}$ was performed also for $\beta$-delayed fission of ${ }^{188} \mathrm{~Pb}$ and ${ }^{196} \mathrm{Po}$ [41], with results similar both to conclusions of the original work and to results of the analysis of the mercury isotopes presented here. 
The magnitude and range of the necessary reduction of fission-barrier height and its interplay with various implementations of pairing suggests that, despite significant uncertainty due to the unknown value of the pairing gap of even-even daughter nuclei at the saddle, its dominant part originates from causes other than the pairing. Such a conclusion is supported by similar observed reduction factors to full fission barriers of nuclei close and far off the neutron shell closure $N=126$ [42,43], where specifically the absolute values of the reduction for nuclei around $N=126$ significantly exceed the magnitude of the pairing gap. Furthermore, it is worth mentioning that the reduction of the full fission barriers required in the statistical-model calculation, specifically in the channels with evaporation of neutrons only, is equal for evaporation residues with both even and odd atomic numbers, and especially for the nuclei with odd proton numbers, where the uncertainty concerning the magnitude of pairing gap does not play a crucial role. To judge the effect of the neutron deficit, the known cases of $\beta$-delayed fission in the in the Am-Es region were also examined (detailed discussion will appear elsewhere [44]). To circumvent the question of the correct choice of pairing gap at the saddle point, the above mentioned variant $\mathrm{D}$ of the calculations was used. In the few cases where spontaneous fission is observed in the ground state, the decay rate was used to estimate the curvature of a parabolic fission barrier, and this value was used to extract the fission probability at sub-barrier energies using the Hill-Wheeler formula. Apart from the nuclei where the extracted fission barriers are close to $Q_{\text {EC }}$, reasonable agreement between the experimental fission barriers and the values calculated using macroscopic fission barriers of Sierk [15] with ground-state shell corrections(see above) is reached, in accordance with the results for nuclear reactions with nuclei close to the $\beta$-stability line [16]. Thus it appears that the observed reduction of fission-barrier heights can be related to the difference of isospin asymmetry compared to the nuclei close to the $\beta$-stability line.

\section{D. "Self-consistent" calculation of $\boldsymbol{P}_{\beta \mathrm{df}}$ for ${ }^{180} \mathrm{Tl}$}

The $\beta$-delayed fission of ${ }^{180} \mathrm{Tl}$ is remarkable because the observed fission fragment distribution is mass asymmetric [19]. It is the first case of asymmetric fission which can not be plausibly related to the shell structure of fragments. The model of fission developed by Möller et al. [45], which calculates the fission potential energy as a function of five shape parameters, suggests that the asymmetric fission is mainly due to local structure of the potential in the saddle region. It thus appears reasonable to try to estimate the probability of $\beta$-delayed fission using the value of the fission-barrier height adopted from the five-dimensional model, which gives a value of 9.81 $\mathrm{MeV}$ for the asymmetric saddle. Also the other components of the model will be taken from the calculations of Möller et al., the ground-state properties from the work [32] (leading to $Q_{\mathrm{EC}}=10.44 \mathrm{MeV}$ ), and the two calculated $\beta$-strength functions, mentioned above. The level density in the saddle configuration is determined using the Fermi-gas formula with the level-density parameter estimated as $a_{\mathrm{f}} \approx A / 13 \mathrm{MeV}^{-1}$, in accordance with the Fermi-gas formula fit of recently calculated level density at the saddle [33]. Thus one can estimate the
TABLE II. Probabilities of $\beta$-delayed fission of ${ }^{180} \mathrm{Tl}$, estimated using "self-consistent" approach; see the text for details.

\begin{tabular}{lr}
\hline \hline$\beta$-strength function & $P_{\beta \mathrm{df}}(\operatorname{Var} . \mathrm{B})$ \\
\hline [34]-p.d. & $3.7 \times 10^{-9}$ \\
[34]-d.d. & $2 \times 10^{-8}$ \\
\hline \hline
\end{tabular}

probability of $\beta$-delayed fission in a "self-consistent" way and avoid uncertainty resulting from the use of model assumptions for specific components from different approaches.

The results of such calculations are given in Table II. The pairing gap in the calculation of the level density at the saddle point was assumed zero, whereas for the initial state it was assumed to have the usual value for the ground state, in accordance with variant $B$. The estimated values of the $\beta$-delayed fission probability are by three to four orders of magnitude smaller than the measured value of $3.2(2) \times 10^{-5}$.

Since the value of $Q_{\mathrm{EC}}$ used is larger than the value for fission-barrier height, tunneling effects, which are not considered in the version of the statistical model used, cannot explain this disagreement. The use of a nonzero value of the pairing gap at the saddle point would further reduce the values of $P_{\beta \mathrm{df}}$. When, instead of a zero value, the full pairing gap is assumed, as it is in variant A, the process becomes deeply sub-barrier. Since the level-density parameter at the saddle point is approximated using the results of the fit from Ref. [33], the value of the level-density parameter was varied by $\pm 50 \%$ and only weak dependence of the results was observed.

Among other model parameters, the experimental value of $P_{\beta \text { df }}$ can be reproduced only by adjusting the minimal energy of excited levels populated in the $\beta$ decay, and thus in effect altering the shape of the $\beta$-strength function. However, the resulting value of $E_{\min }=9-9.5 \mathrm{MeV}$ (variation reflects the choice of $\beta$-strength function) would signal a very unusual behavior where only very high energy levels are populated. Thus, it appears that a statistical model employing the "selfconsistent" parameter values is not capable of reproducing the experimental $P_{\beta \text { df }}$ for ${ }^{180} \mathrm{Tl}$.

To reproduce this value, it is necessary to adjust the fission-barrier height, as it is done in the previous subsection (see Table I). It is in principle possible that the necessity to reduce the fission barrier follows from some inconsistency in the statistical model, which uses rather a schematic description of the fission decay. Nevertheless, for heavier nuclei in the Am-Es region the statistical model allows us to reproduce experimental probabilities of $\beta$-delayed fission reasonably well with the rotating-liquid-drop models of Cohen, Plasil, and Swiatecki [14] and Sierk [15], which are routinely used in evaporation-residue cross-sectional calculations, so the use of a statistical model appears justified.

\section{SUMMARY AND CONCLUSIONS}

In summary, the fission-barrier height was deduced for ${ }^{180} \mathrm{Hg}$ using the experimentally determined probability of $\beta$ delayed fission of ${ }^{180} \mathrm{Tl}$. Four alternative $\beta$-strength functions 
and four variants of statistical calculations were considered. Depending upon the model, the deduced fission barriers appear to be $10-40 \%$ smaller than theoretical estimates. This is consistent with the earlier results obtained using the statistical model of de-excitation of compound nuclei. This observation was confirmed for the $\beta$-delayed fission of ${ }^{178} \mathrm{Tl}$ as well, albeit using only a flat $\beta$-strength function. At the same time, fission barriers extracted from $P_{\beta \text { df }}$ of nuclei, which lie closer to the $\beta$-stability line (observed in the region of heavier transuranium nuclei), using the same procedures appear to be consistent with theoretical values. Thus it appears that the observed reduction of fission-barrier heights can be related to the difference of isospin asymmetry compared to the nuclei close to the $\beta$-stability line. Reduction of fission barriers obtained using our analysis of the probability of $\beta$-delayed fission of neutron-deficient nuclei is in agreement with the values extracted from evaporation-residue cross sections. However, a part of the observed reduction of fission barriers in $\beta$-delayed fission can be attributed to uncertainty of the magnitude of the pairing gap in the saddle point of the even-even daughter nucleus. In this sense, the fact that the reduction of fission barriers corresponding to observed evaporation-residue cross sections is identical for products with both even and odd atomic numbers suggests that the main part of the reduction of the fission barrier is caused by other reasons than pairing interaction. Nevertheless, such conclusion needs to be verified using the fission of odd-odd nuclei, which are not accessible via $\beta$-delayed fission. In this respect, low-energy fission induced by transfer reactions of postaccelerated radioactive beams offers unique possibilities to obtain unambiguous conclusions; see the discussion in [46]. Future facilities like, for example, HIE-ISOLDE at CERN will provide radioactive beams with sufficient energy and intensity for such studies.

\section{ACKNOWLEDGMENTS}

This work has been supported by the Slovak Scientific Grant Agency under Contracts No. 2/0105/11 and No. 1/0613/11, by the Slovak Research and Development Agency under Contracts No. APVV-0177-11 and No. APVV-0105-10, by the UK Science and Technology Facilities Council Grant No. ST/H008691/1, by FWO-Vlaanderen (Belgium), GOA (BOF-KU Leuven), and the Interuniversity Attraction Poles Programme, Belgian State, Belgian Science Policy (BriX Network P6/23), by Reimei Foundation of JAEA, and by DAIWA Anglo-Japanese Foundation. P.M. and A.J.S. acknowledge that this work was carried out under the auspices of the National Nuclear Security Administration of the US Department of Energy at Los Alamos National Laboratory under Contract No. DE-AC52-06NA25396 and the US Department of Energy through the LANL/LDRD Program.
[1] C. Wagemans, The Nuclear Fission Process (CRC Press, Boca Raton, FL, 1991), Chap. 12, p. 580.

[2] S. Bjornholm and J. E. Lynn, Rev. Mod. Phys. 52, 725 (1980).

[3] M. Beckerman and M. Blann, Phys. Rev. C 17, 1615 (1978).

[4] A. Gavron, Phys. Rev. C 21, 230 (1980).

[5] W. Reisdorf et al., Z. Phys. A 300, 227 (1981).

[6] D. Vermeulen et al., Z. Phys. A 318, 157 (1984).

[7] C. Sahm et al., Nucl. Phys. A 441, 316 (1985).

[8] W. Reisdorf et al., Nucl. Phys. A 444, 154 (1985).

[9] A. N. Andreyev et al., Yad. Fiz. 52, 640 (1990) [Sov. J. Nucl. Phys. 52, 412 (1990)].

[10] A. N. Andreyev et al., Nucl. Phys. A 620, 229 (1997).

[11] A. N. Andreyev et al., Phys. Rev. C 72, 014612 (2005).

[12] R. N. Sagaidak and A. N. Andreyev, Phys. Rev. C 79, 054613 (2009).

[13] W. D. Myers and W. J. Swiatecki, Ark. Fys. 36, 343 (1967); Proceedings of the Lysekil Symposium: Nuclides far off the Stability Line, Aug. 21-27, 1966, Sweden.

[14] S. Cohen, F. Plasil, and W. J. Swiatecki, Ann. Phys. 82, 557 (1974).

[15] A. J. Sierk, Phys. Rev. C 33, 2039 (1986).

[16] A. V. Ignatyuk et al., Yad. Fiz. 21, 1185 (1975) [Sov. J. Nucl. Phys. 21, 612 (1976)].

[17] V. I. Kuznecov, N. K. Skobelev, and G. N. Flerov, Yad. Fiz. 4, 99 (1966) [Sov. J. Nucl. Phys. 4, 70 (1967)].

[18] E. Ye. Berlovich and Yu. N. Novikov, Phys. Lett. B 29, 155 (1969).

[19] A. N. Andreyev et al., Phys. Rev. Lett. 105, 252502 (2010).

[20] H. V. Klapdor, Prog. Part. Nucl. Phys. 10, 131 (1983).
[21] G. Audi and M. Wang (private communication).

[22] N. Bohr and J. Wheeler, Phys. Rev. 56, 426 (1939).

[23] A. V. Ignatyuk, G. N. Smirenkin, and A. N. Tishin, Yad. Fiz. 21, 485 (1975) [Sov. J. Nucl. Phys. 21, 255 (1975)].

[24] A. Stolovy and J. A. Harvey, Phys. Rev. 108, 353 (1957).

[25] A. Gilbert and A. G. W. Cameron, Can. J. Phys. 43, 1446 (1965).

[26] J. Elseviers et al. (unpublished).

[27] V. Liberati et al. (unpublished).

[28] A. Staudt, M. Hirsch, K. Muto, and H. V. KlapdorKleingrothaus, Phys. Rev. Lett. 65, 1543 (1990).

[29] Yu. A. Lazarev et al., Europhys. Lett. 4, 893 (1987).

[30] Yu. A. Lazarev et al., Proc. Int. School-Seminar on Heavy Ion Physics, Dubna 1989, report D7-90-142 (JINR, Dubna, 1990), p. 208.

[31] Yu. A. Lazarev et al., Proc. 6th Int. Conf on Nuclei Far From Stability \& 9th Int. Conf. on Atomic Masses and Fundamental Constants, Bernkastel-Kues, Germany, 1992, Inst. Phys. Conf. Ser. 132 (IOP Publishing, Bristol, 1993) p. 739.

[32] P. Möller, J. R. Nix, W. D. Myers, and W. J. Swiatecki, At. Data Nucl. Data Tables 59, 185 (1995).

[33] P. Möller, A. J. Sierk, T. Ichikawa, A. Iwamoto, R. Bengtsson, H. Uhrenholt, and S. Åberg, Phys. Rev. C 79, 064304 (2009).

[34] P. Möller and J. Randrup, Nucl. Phys. A 514, 1 (1990).

[35] J. Elseviers et al., Phys. Rev. C 84, 034307 (2011).

[36] M. Seliverstov et al. (unpublished).

[37] P. Möller, A. J. Sierk, R. Bengtsson, H. Sagawa, and T. Ichikawa, Phys. Rev. Lett. 103, 212501 (2009).

[38] T. Grahn et al., Phys. Rev. Lett. 97, 062501 (2006). 
[39] P. Möller and J. R. Nix, At. Data Nucl. Data Tables 26, 165 (1981).

[40] G. Audi, A. H. Wapstra, and C. Thibault, Nucl. Phys. A 729, 129 (2003).

[41] A. N. Andreyev et al., Phys. Lett. B 312, 49 (1993).

[42] D. D. Bogdanov et al., Yad. Fiz. 62, 1931 (1999) [Phys. At. Nucl. 62, 1794 (1999)].

[43] M. Veselsky et al., arXiv:nucl-ex/0403001; Proceedings of the International Conference on Dynamical Aspects of
Nuclear Fission, Casta-Papiernicka, Slovakia, 1996, edited by J. Kliman and B. I. Pustylnik (JINR, Dubna, 1996), p. 129.

[44] M. Veselsky et al. (unpublished).

[45] P. Möller, D. G. Madland, A. J. Sierk, and A. Iwamoto, Nature (London) 409, 785 (2001).

[46] M. Veselsky et al., Transfer-induced fission of heavy radioactive beams, Letter of Intent CERN-INTC-2010-026/I-095, CERN, Geneva, 2010 . 